\title{
TWO UC-SETS WHOSE UNION IS NOT A UC-SET
}

\author{
JOHN J. F. FOURNIER ${ }^{1}$
}

\begin{abstract}
It is shown that the union of two sets of uniform convergence need not be a set of uniform convergence.
\end{abstract}

We use the standard terminology of harmonic analysis on the unit circle as in [4]. We recall some notions discussed in [8] and [9], and in the references cited in these papers.

Definition. Given a subset $E$ of the integers, call an integrable function $f$, on the circle, an $E$-function if $\hat{f}(n)=0$ for all integers $n$ outside $E$, and denote the space of continuous $E$-functions by $C_{E}$. Call $E$ a set of uniform convergence, or $a U C$-set, if every function in $C_{E}$ has a uniformly convergent Fourier series.

The union problem for UC-sets is mentioned as an open problem in $[5$, p. 86; 9 , p. 283]. To solve it, we need a few more facts about UC-sets. It is known that $E$ is a UC-set if and only if there is a constant $\kappa$ so that, for each function $f$ in $C_{E}$, the partial sums $S_{N}(f)$ of the Fourier series of $f$ satisfy the inequality $\left\|S_{N}(f)\right\|_{\infty} \leqslant$ $\kappa\|f\|_{\infty}$ for all nonnegative integers $N$. Furthermore, when $E$ is a UC-set, there is a smallest value of $\kappa$ for which the inequality above holds for all such $f$ and $N$; this smallest value of $\kappa$ is called the $U C$-constant of $E$, and is denoted by $\kappa(E)$. If $E$ is a UC-set, then so is every translate of $E$, but it turns out that the translates of a UC-set do not all have to have the same UC-constant.

DEFINITION. Call $E$ a $C U C$-set, or a set of completely uniform convergence if $E$ is a UC-set with the additional property that the sequence $\{\kappa(E+n)\}_{n=-\infty}^{\infty}$ is bounded.

This notion was introduced, independently by G. Travaglini [9, Lemma 6] and F. Ricci [7, p. 426]. In [8], P. M. Soardi and Travaglini gave some nontrivial examples of CUC-sets, and they showed that if there is a UC-set that is not a CUC-set, then there is a pair of UC-sets whose union is not a UC-set. In the present paper, we exhibit a class of UC-sets that are not CUC-sets, thereby showing that the union of two UC-sets need not be a UC-set.

Recall that a set $H$ of positive integers is called a Hadamard set if there is a constant $r>1$ so that, when $H$ is enumerated in increasing order as $\left\{h_{j}\right\}_{j=1}^{J}$, then $h_{j+1} \geqslant r h_{j}$ for all $j$. Also, if $E$ and $F$ are two sets of integers then $E-F$ denotes the set of all integers of the form $m-n$ where $m \in E$ and $n \in F$.

Received by the editors March 2, 1981.

1980 Mathematics Subject Classification. Primary 42A55.

${ }^{1}$ Research partially supported by Canadian N.S.E.R.C. operating grant number A-4822.

(c) 1982 American Mathematical Society $0002-9939 / 82 / 0000-0016 / \$ 02.00$ 
ThEOREM. Let $H$ be an infinite Hadamard set. Then $H-H$ is a UC-set, but it is not a CUC-set.

Proof. Let $E=H-H$. To show that $E$ is a UC-set, it suffices, by [9, Theorem 2], to show that the positive and negative parts of $E$ are both UC-sets. Since $E$ is symmetric, it is enough to do this for the positive part of $E$. Finally, by [9, Theorem 3], it is enough to show that

$$
\sup _{N>0} \kappa(E \cap[N, 2 N])<\infty .
$$

To this end, enumerate $H$ in increasing order as $\left\{h_{j}\right\}_{j=1}^{\infty}$, and let $r>1$ be as in the definition of Hadamard set. Fix a positive integer $N$, and consider the indices $j$ for which, for some index $i<j$, the difference $h_{j}-h_{i}$ lies in the interval $[N, 2 N]$. Let $J$ be the smallest such index $j$; then $h_{J}>N$. On the other hand, if $j$ is any such index, then, in particular,

$$
2 N>h_{j}-h_{j-1}>(r-1) h_{j-1}>(r-1) r^{j-1-J} h_{J}>(r-1) r^{j-J-1} N .
$$

Thus, $j-J-1<\log [2 /(r-1)] / \log r=L(r)$, say. It follows that there are at most $L(r)+1$ such indices $j$, and hence that $E \cap[N, 2 N]$ is included in the union of at most $L(r)+1$ translates of the set $-H$. Therefore there is a constant $C(r)$ so that $E \cap[N, 2 N]$ has Sidon constant at most $C(r)$, and $\kappa(E \cap[N, 2 N])<C(r)$ also. Thus, $E$ is indeed a UC-set.

To see that $E$ is not a CUC-set, fix a positive integer $M$, and consider the Hilbert matrix $\left\{A_{m, n}\right\}_{m, n=1}^{M}$ given by letting

$$
A_{m, n}= \begin{cases}0 & \text { if } m=n \\ \frac{1}{m-n} & \text { otherwise. }\end{cases}
$$

Recall [3, Example 5.7] that the norm of $A$, as an operator on $l^{2}$, is at most $\pi$. Given a number $\theta$ in the interval $[0,2 \pi)$, let $v(\theta)$ be the vector in $C^{M}$ with $j$ th component $v_{j}(\theta)=\exp \left(i h_{j} \theta\right)$ for all $j$, and let

$$
f(\theta)=(v(\theta), A v(\theta))=\sum_{m, n=1, m \neq n}^{M} \frac{1}{m-n} \exp \left[i\left(h_{m}-h_{n}\right) \theta\right] .
$$

Then $f$ is an $(H-H)$-polynomial. Moreover,

$$
|f(\theta)|<\|A\|\left(\|v(\theta)\|_{2}\right)^{2}<\pi M
$$

for all $\theta$, so that $\|f\|_{\infty}<\pi M$. On the other hand,

$$
\begin{aligned}
\sum_{k>0} \hat{f}(k) & =\sum_{1<n<m<M} \frac{1}{m-n}=\sum_{j=1}^{M-1}(M-j) \frac{1}{j} \\
& =(M-1)+M\left(\sum_{j=2}^{M-1} \frac{1}{j}\right)-\sum_{j=2}^{M-1} 1 \\
& >M(\log M-\log 2)>(1 / \pi)\|f\|_{\infty} \log (M / 2)
\end{aligned}
$$


Let $N=h_{M}$, and let $g(\theta)=f(\theta) \exp (-i N \theta)$. Then $g$ is an $\left(E-h_{M}\right)$-polynomial, and

$$
\left\|S_{N}(g)\right\|_{\infty}>\left|\sum_{|n|<N} \hat{g}(n)\right|=\sum_{k>0} \hat{f}(k)>(1 / \pi)\|g\|_{\infty} \log (M / 2) .
$$

Therefore, $\kappa\left(E-h_{M}\right)>(1 / \pi) \log (M / 2)$ for all $M$, and $E$ is not a CUC-set. See Remark 3 below for another proof that $E$ is not a CUC-set.

REMARK. 1. Now that we have examples of UC-sets that are not CUC-sets, we can, as pointed out in [8] easily construct pairs of UC-sets whose union is not a UC-set. Indeed, let $H=\left\{h_{j}\right\}_{j=1}^{\infty}$ be a Hadamard set for which in fact $h_{j+1} \geqslant 2 h_{j}$ for all $j$; given $H$, let

$$
A=\left\{m: m=h_{i}-h_{j}+h_{k} \text { where } i>j>k\right\} .
$$

Then, by the proof of Proposition 2 of [8], the sets $A$ and $B$ are both UC-sets, but $A \cup B$ is not a UC-set.

2. A related example is suggested by an observation on p. 283 of [9]. Suppose that, in the example above, the integers $h_{j}$ are all even, and let $C=A \cup(B-1)$. Then $C$ is a UC-set, as is $C+1$, but $C \cup(C+1)$ is not a UC-set, because it includes $A \cup B$.

3. The second part of the proof of our theorem actually shows that if $E$ and $F$ are two infinite sets of positive integers, then $E-F$ is not a CUC-set. Here is an amusing alternate proof of this implication. If $E-F$ were a CUC-set, then, by [8, Proposition 1], there would exist a measure $\mu$ such that

$$
\hat{\mu}(n)= \begin{cases}1 & \text { if } n \in E-F \text { and } n>0 \\ 0 & \text { if } n \in E-F \text { and } n<0 .\end{cases}
$$

Enumerate the sets $E$ and $F$ as $\left\{m_{j}\right\}_{j=1}^{\infty}$ and $\left\{n_{j}\right\}_{j=1}^{\infty}$ respectively, and, for each index $j$, let $\phi_{j}$ and $\psi_{j}$ be the functions on $[0,2 \pi)$ given respectively by $t \mapsto$ $\exp \left(-i m_{j} t\right)$ and $t \mapsto \exp \left(+i n_{j} t\right)$; then

$$
\int_{T} \phi_{j}(t) \psi_{k}(t) d \mu(t)=\hat{\mu}\left(m_{j}-n_{k}\right)= \begin{cases}1 & \text { if } m_{j}>n_{k i}, \\ 0 & \text { if } m_{j}<n_{k} .\end{cases}
$$

Let $\phi$ and $\psi$ be accumulation points in $L^{\infty}(d|\mu|)$ of the respective sequences $\{\phi\}_{j=1}^{\infty}$ and $\left\{\psi_{k}\right\}_{k=1}^{\infty}$. Then $\int \phi \psi d \mu$ can be approximated arbitrarily well by integrals of the form $\int \phi \psi_{k} d \mu$, and any such integral can in turn be approximated arbitrarily well by integrals of the form $\int \phi_{j} \psi_{k} d \mu$, where $m_{j}>n_{k}$; hence $\int \phi \psi d \mu=1$. On the other hand, by approximating $\phi$ first by $\phi_{j}$, and then approximating $\psi$ by $\psi_{k}$, where $n_{k}>m_{j}$, one sees that $\int \phi \psi d \mu$ must also be equal to 0 . This contradiction shows that there is no such measure $\mu$, and hence that $E-F$ is not a CUC-set.

4. It follows from the implication above that, if $E, F$, and $G$ are infinite sets of positive integers, then $E-F+G$ and $E-F-G$ are not UC-sets. This contrasts with the fact [9, Theorem 7] that, if $E$ is a Paley set, in other words a union of finitely-many Hadamard sets, then $E+E+E$ is a UC-set, as is $E+E+E+E$, etc. In view of our main theorem, one might ask if $E-E$ must be a UC-set whenever $E$ is a Paley set; the answer is "no", because there are pairs $\left(E_{1}, E_{2}\right)$ of Hadamard sets for which $E_{1}-E_{2}$ consists of all integers [5, p. 69]. In a similar 
vein, one can ask $[9$, p. 283] whether $E+E$ must be a UC-set whenever $E$ is a dissociate set of positive integers; see $[5$, p. 19] for a definition of "dissociate". The answer is again "no"; the proof uses Hilbert matrices, and will appear in [2].

5. It is known [9, Lemma 6] that subsets of the positive integers that are UC-sets are also CUC-sets. Therefore, the sets $A$ and $B$ considered in Remark 1 provide an example of a pair of CUC-sets whose union is not even a UC-set.

6. Fix a strictly increasing sequence $N=\left\{N_{j}\right\}_{j=1}^{\infty}$ of positive integers, and call a set $E$ a $U C(N)$-set if for every function $f$ in $C_{E}$, the sequence $\left\{S_{N_{j}}(f)\right\}_{j=1}^{\infty}$ converges uniformly. It was pointed out by B.-Y. $\mathrm{Ng}$ [6] that when $N=\left\{2^{j}\right\}_{j=1}^{\infty}$ there are pairs of $\mathrm{UC}(N)$-sets whose union is not a $\mathrm{UC}(N)$-set. In fact, the methods of the present paper show that, for each such strictly increasing sequence $N$, there is a pair of CÚC-sets $A$ and $B$, as in Remark 1, whose union is not a UC(N)-set.

7. Given an index $p$ in the interval $[1, \infty)$, and a set $E$ of integers, let $L_{E}^{p}$ be the subspace of all $E$-functions in $L^{p}(T)$. Call $E$ an $L^{p} C$-set if $\left\|S_{N}(f)-f\right\|_{p} \rightarrow 0$ as $n \rightarrow \infty$ for all $f$ in $L_{E}^{p}$. Again, $E$ is an $L^{p} C$-set if and only if the quantity $\kappa_{p}(E)=\sup _{f, N}\left\{\left\|S_{N}(f)\right\|_{p}: f \in L_{E}^{p},\|f\|_{p}=1, N\right.$ a nonnegative integer $\}$ is finite. Finally, call $E$ a $C L^{p} C$-set if the sequence $\left\{\kappa_{p}(E+n)\right\}_{n=-\infty}^{\infty}$ is bounded. These notions are not interesting when $1<p<\infty$, because the M. Riesz theorem shows that every set is a $C L^{p} C$-set in that case. It is not known, however, whether the classes of $L^{1} C$-sets or $C L^{1} C$-sets are closed under finite unions. S. Hartman [private communication] has observed that our examples shed some light on the relations between these classes and the classes of UC-sets and CUC-sets. First, it is easy to see that every UC-set is an $L^{1} C$-set, and that every CUC-set is a $C L^{1} C$-set. The examples given in Remark 1 show that the converses to these implications are false. Indeed, it is known [1, Theorem 5] that the set $A \cup B$ is a $\Lambda(2)$-set; it follows that $A \cup B$ is a $C L^{1} C$-set although it is not a UC-set.

8. I am pleased to acknowledge that the main theorem of this paper resulted from conversations with Ron Blei and Gordon Woodward. I have also benefited from helpful comments by S. Hartman, E. Sawyer, P. M. Soardi, and G. Travaglini.

\section{REFERENCES}

1. A. Bonami, Etude des coefficients de Fourier de fonctions de $L^{P}(G)$, Ann. Inst. Fourier (Grenoble) 20 (1970), 335-402. MR 44 \# 727.

2. J. J. F. Fournier and L. Pigno, Analytic and arithmetic properties of thin sets (to appear).

3. P. R. Halmos and V. S. Sunder, Bounded integral operators on $L^{2}$ spaces, Ergebnisse der Math. und ihrer Grenzgebiete, Vol. 96, Springer-Verlag, Berlin and New York, 1978.

4. Y. Katznelson, An introduction to harmonic analysis, Wiley, New York, 1968.

5. J. M. López and K. A. Ross, Sidon sets, Lecture Notes in Pure and Appl. Mathematics, Vol. 13, Marcel Dekker, New York, 1975.

6. B.-Y. Ng, Union of UC-sets, Abstracts Amer. Math. Soc. 1 (1980), 171.

7. F. Ricci, A multiplicative structure on some spaces of pseudomeasures on the circle and related properties, Bull. Sci. Math. (2) 103 (1979), 423-434.

8. P. M. Soardi and G. Travaglini, On sets of completely uniform convergence, Colloq. Math. (to appear).

9. G. Travaglini, Some properties of UC-sets, Boll. Un. Mat. Ital. B (5) 15 (1978), 272-284.

Department of Mathematics, University of British Columbia, Vancouver, Canada V6T 1Y4 